\title{
Valutazione comparativa dell'analisi biochimica e microbiologica dell'urina raccolta convenzionalmente e con un nuovo metodo
}

\author{
Sergio Frugoni, Adriana Ripamonti', Federica Di Berardino², Cristina Di Berardino ${ }^{3}$ \\ Laboratorio Analisi Chimico Cliniche e Microbiologiche, Istituto Geriatrico Pio Albergo Trivulzio, Milano \\ Laboratorio analisi Milano, Buccinasco, Milano \\ 2 Pediatra di base ASL Vimodrone, Milano \\ ${ }^{3}$ Medicina generale ASL Milano
}

\section{Comparative biochemical and microbiological urine analysis with conventional and new collection methods}

Key words: Urine, New collection methods, Microbiological and biochemical analysis

\section{SUMMARY}

Although urinary tract infection (UTI) is common in childhood, it continues to be underdiagnosed, despite its association with renal failure (if the infection is not treated promptly, bacteria may then go up the ureters to infect the kidneys: pyelonephritis). This is partly due to the fact that urine samples are often difficult to obtain. A new infant urine collection kit (Pronturina ${ }^{\circledR}$ ) is now available. This consists of an absorbent panty-liner which is placed inside the nappy. Once wet it is removed and inserted into the syringe provided. The urine is then squeezed into the tube used to take the specimen to the doctor's surgery.

Tests have been carried out to prove that this new method for urine collection does not change the diagnostically valuable markers or bacterial culture parameters associated with conventional chemical and microbiological urine analysis. The specimens can be tested and examined up to six hours after collection. When microscopic sediment examination and culture are considered necessary, as follow-up to leukocyte esterase and nitrate examination, specimens are sent to the laboratory. A tablet-form stabilizing agent can be added to the sample in order to obtain a urinalysis delay time of I day at room temperature. Comparative results showed no significant differences between the various methods of collection and preservation.

This easy new method is particularly suitable for urine collection in infants and could change the approach to UTI diagnosis.

\section{INTRODUZIONE}

L'esame delle urinee nel lattante e nell'adulto non collaborante è una richiesta diagnostica frequente. Rappresenta uno strumento importante per escludere l'infezione delle vie urinarie (IVU) quando il paziente manifesta febbre senza altri sintomi specifici di malattia.

L'IVU nell'infanzia non è facile da diagnosticare, e ancora oggi è talvolta misconosciuta nonostante possa determinare danni renali. Un risultato alterato di un qualsiasi parametro dell'esame chimicofisico dell'urina deve far sospettare la possibilità di IVU (4). È utile pertanto eseguire lo stick urine considerando i diversi parametri. La ragionevole esclusione di IVU è ottenuta soltanto se si effettua l'esame microscopico del sedimento $(1,2,3)$.

Il dosaggio a fresco con semplice striscia reattiva dell'esterasi leucocitaria e/o dei nitriti su urine non raccolte sterilmente fornisce una probabilità posttest sufficientemente buona ed accurata per poter prendere una decisione clinica ragionevolmente fondata (8). La sensibilità e la specificità della determinazione dell'esterasi leucocitaria e/o dei nitriti è rispettivamente dell' $85 \%$ e dell' $80 \%(7)$.

Poiché non è facile raccogliere le urine dei bam- bini e degli adulti non collaboranti spesso questo semplice esame non è eseguito. I metodi attuali non invasivi sono indaginosi e non facilmente eseguibili e generano il disuso dell'esame ambulatoriale e il ricorso alle strutture ospedaliere con un grande onere di tempo ed economico sia dei pazienti sia degli operatori sanitari.

Attualmente è disponibile un nuovo semplice sistema per la raccolta dell'urina, Pronturina ${ }^{\circledR}$ (Promesan, Milano, Italia), il metodo, basato sulla spremitura di un assorbente, può essere utilizzato da persona non specializzata anche a casa del bambino. La provetta, contenente il campione d'urina, potrà essere portata sia al medico curante sia al laboratorio d'analisi. Tali condizioni potrebbero far superare le attuali difficoltà diagnostiche e consentire il trattamento adeguato delle IVU. Ancora oggi si discute sulla gestione del bambino con febbre ed assenza di sintomi specifici e sull'utilità dell'esame chimico-fisico delle urine nei confronti dell'urinocoltura (gold standard diagnostico) per confermare o escludere la diagnosi di IVU.

Scopo del lavoro è stato quello di verificare 1) l'interferenza delle modalità di raccolta (assorbente ed il tempo) sui parametri analitici, 2) la 
possibilità di utilizzare un conservante per consegnare il campione al laboratorio evitando di ripetere la raccolta.

\section{MATERIALI E METODI}

Lo schema dello studio è sintetizzato in figura I, sono stati presi in esame 50 campioni d'urine provenienti da pazienti collaboranti affetti da IVU (preselezionati sulla contemporanea presenza di esterasi leucocitaria e nitriti nelle urine). Al fine di valutare se la procedura di simulazione della raccolta potesse contaminare $i$ campioni sono stati analizzati anche 16 urine provenienti da pazienti sani (anamnesi e stick urine negative). I campioni sono stati raccolti da mitto intermedio in contenitore sterile. Da ciascun campione è stata prelevata un aliquota di $10 \mathrm{ml}$ che è stata depositata sull'assorbente contenuto nel kit Pronturina ${ }^{\circledR}$. Dopo un'ora a temperatura ambiente $\left(26^{\circ} \mathrm{C}, 45 \%\right.$ U.R.) l'urina è stata recuperata dall'assorbente seguendo le istruzioni allegate al kit: l'assorbente è stato arrotolato ed inserito nel cilindro di una siringa priva di pistone, reinserito il pistone è stato spremuto l'assorbente raccogliendo l'urina in due provette sterili. Una è stata conservata a temperatura ambiente per 4 ore (gruppo "Pronturina"), l'altra, contenente una compressa di stabilizzante (Cult-Ur 10, Promesan, Milano Italia), conservata sempre a temperatura ambiente per 24 ore (gruppo "Stabilizzante"). L'esame dell'urina e l'urinocoltura sono stati eseguiti con tutti i campioni: subito sui campioni originali (partenza) ed ai tempi predefiniti nel gruppo "Pronturina" e "Stabilizzante".

\section{Esame chimico fisico:}

I parametri chimici sono stati determinati utilizzando strisce con aree di reazione (Urisys 2400 Cassette, Roche), la lettura è stata effettuata mediante strumentazione automatica Urisys 2400 (Roche).

\section{Esame colturale:}

\section{Terreni utilizzati}

L'esame colturale è stato eseguito inoculando terreni agarizzati CLED e Mac Conckey. CLED agar è stato utilizzato per la conta totale di Unità Formanti Colonia (UFC), e preferito a TSA agar per l'inibizione della sciamatura di P.mirabilis che, eventualmente presente, avrebbe impedito una corretta valutazione della conta batterica totale.

\section{Tecniche di semina}

I campioni di urina sono stati diluiti una prima volta 1: 10 con soluzione fisiologica sterile; 100 $\mu \mathrm{L}$ della diluizione sono stati inoculati in CLED agar (piastra I). $1 \mathrm{~mL}$ è stato ulteriormente diluito $1: 10$, sempre in soluzione fisiologica sterile, in modo tale da raggiungere una diluizione finale dei campioni di $1: 100 ; 10 \mu \mathrm{L}$ della diluizione sono stati inocula ti in CLED agar (piastra II).

Le piastre di Mac Conckey agar sono state inoculate direttamente con $1 \mu \mathrm{L}$ di campione di urina.

Le piastre sono state incubate per una notte a $35^{\circ} \mathrm{C}$ in termostato ad aria.

Il processo analitico descritto è stato utilizzato sia per i campioni di urina da mitto intermedio che per quelli ottenuti da spremitura dell'assorbente.

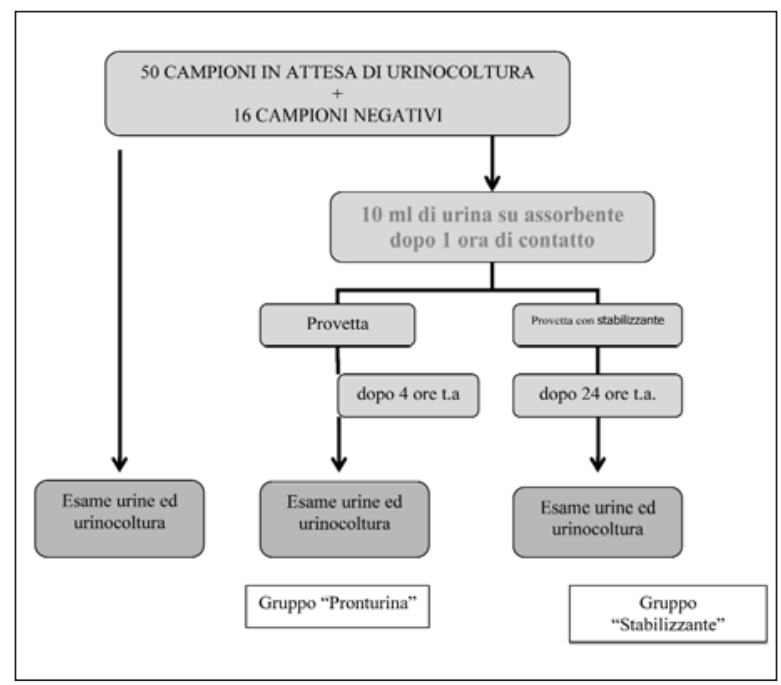

Figura I. Programma della verifica

\section{Conta batterica}

La carica batterica totale è stata determinata confrontando e valutando le UFC presenti nelle piastre I e II.

Il numero di UFC presenti nella piastra I è stato moltiplicato per 100 .

Il numero di UFC presenti nella piastra II è stato moltiplicato per 10000 .

La carica batterica totale è stata espressa in $\mathrm{UFC} / \mathrm{mL}$.

\section{Analisi statistica:}

I risultati del confronto tra i gruppi sono stati valutati mediante grado di correlazione lineare mediante Rho di Spearman e analisi non parametrica (Test di Wilcoxon).

\section{RISULTATI}

In nessun campione si è osservata una differenza significativa della carica batterica totale determinata in piastra I e la carica batterica totale determinato in piastra II.

La carica batterica totale è rimasta la stessa sia nel campione di prima semina (sterile), sia nel campione del gruppo "Prontourina", sia nel campione del gruppo "Stabilizzante". Questo risultato si è osservato in tutti i 50 campioni esaminati.

Non si sono evidenziate differenze significative 
Tabella I. Confronto dei parametri analizzati (esame urine) nei tre gruppi

\begin{tabular}{|c|c|c|c|c|c|}
\hline & $\begin{array}{c}\text { STERILE } \\
(\text { MEDIA } \pm \text { D.S.) }\end{array}$ & $\begin{array}{l}\text { PRONTURINA } \\
\text { (MEDIA } \pm \text { D.S.) }\end{array}$ & $\mathbf{P *}$ & $\begin{array}{c}\text { STABILIZZANTE } \\
\text { (MEDIA } \pm \text { D.S.) }\end{array}$ & $\mathbf{P}^{*}$ \\
\hline Leucociti & $237,5 \pm 234,4$ & $277,1 \pm 235,3$ & ns & $129,2 \pm 223,8$ & ns \\
\hline Nitriti & $0,5 \pm 0,5$ & $0,6 \pm 0,5$ & ns & $0,75 \pm 0,45$ & ns \\
\hline Sangue & $123,75 \pm 1 \mid 2,1$ & $125,8 \pm 110,3$ & ns & $123,7 \pm 1 \mid 2,1$ & ns \\
\hline Proteine & $27,1 \pm 31,0$ & $33,3 \pm 32,6$ & ns & $31,2 \pm 33,9$ & ns \\
\hline
\end{tabular}

fra i tre gruppi costituiti con le urine dei pazienti affetti da IVU (tabella 1). Tutti i parametri analizzati nei tre gruppi, riportati in tabella 1, correlavano perfettamente $(r>0,95)$.

Tutti i campioni provenienti dai soggetti sani sono risultati negativi sia all'esame chimico fisico delle urine, sia all'urinocoltura.

\section{DISCUSSIONE}

I nostri risultati confermano la validità del nuovo metodo di raccolta: la persistenza invariata dei parametri analitici presi in esame dopo il contatto con l'assorbente e la possibilità di raccogliere l'urina e di manipolarla con Pronturina ${ }^{\circledR}$ a temperatura ambiente, senza che si realizzi un inquinamento batterico (urinocoltura falsamente positiva). Infatti la modalità di raccolta in condizioni di non sterilità ed il mantenimento del campione per 4 ore a temperatura ambiente non ha condizionato i risultati sia dell'esame dell'urina sia dell'urinocoltura in tutti i campioni analizzati. Risultati opposti, contaminazione batterica elevatissima, sono stati segnalati in letteratura quando l'urina è stata raccolta con il classico sacchetto detto sterile (6).

Queste osservazioni suggeriscono che le procedure di raccolta invasive potrebbero essere riservate ai soli casi particolari e non eseguiti semplicemente per garantire la sterilità del campione. L'urinocoltura è necessaria sia nel caso uno o più parametri dello stick urine risulti positivo, sia se negativo e non si è in grado di eseguire l'esame microscopico del sedimento (5). Nel caso non sia possibile eseguire l'urinocoltura entro 4 ore, la stessa urina potrà essere utilizzata entro 24 ore se si aggiunge lo stabilizzante. Anche in questo caso i risultati sono stati sovrapponibili e concordanti ai precedenti.

Il giudizio del personale tecnico del laboratorio che ha realizzato la verifica è stato positivo: la procedura di raccolta è stata considerata valida e di semplice esecuzione.

Sulla base di queste evidenze precliniche possiamo ipotizzare un protocollo per gestire il bambino con febbre e possibile infezione delle vie urinarie: il pediatra potrà chiedere ai genitori di rac- cogliere l'urina e portarla con il bambino alla visita, eseguirà lo stick urine in studio e se lo riterrà necessario invierà entro 4 ore in laboratorio lo stesso campione, per la conferma diagnostica (esame urine ed urinocoltura). Se l'esame sarà eseguito il giorno dopo, dovrà aggiungere lo stabilizzante.

La fattibilità e la validità di questa proposta dovrà essere verificata clinicamente.

\section{BIBLIOGRAFIA}

1. American Academy of Pediatrics Committee on Quality Improvement. Subcommitte on urinary tract infection. Practice parameters: the diagnosis, treatment, and evaluation of the initial urinary tract infction in febrile infants and young children. Pediatrics 1999; 103: 343-52.

2. Bachur R, Harper RB. Reliability of the urinalysis for predicting urinary tract infection in young febrile children. Arc Pediatr Adolesc Med 2001; 155: 60-5.

3. Bulloch B, Bausher JC, Pomerantz WJ, et al. Can urine clarity exclude the diagnosis of urinary tract infection? Pediatrics 2000; 106: E60.

4. Children's Hospital Medical Center of Cincinnati. Evidence based clinical practice guideline for patients 6 years of age less with a first time acute urinary tract infection (UTI). http://www.guidelines.gov

5. Gorelick MH, Shaw W. Screening tests for urinary tract infection in children: a meta-analysis. Pediatrics 1999; 104: 54.

6. Grisaru-Soen G, Goldman R, Barzilai A, et al. Falsepositive urine cultures using bag collection. Clin Pediatr 2000; 39: 499-500.

7. Huicho L, Campos-Sanchez M, Alamo C. Metaanalysis of urine screening tests for determinino the risk of urinary tract infection in children. Pediatr Infect Dis 2002; 21: 1-11.

8. Sopo SM. È sufficiente l'esame chimico-fisico delle urine per fare diagnosi di infezione delle vie urinarie? Area Pediatrica 2004; 5: 13-6.

\section{Sergio Frugoni}

Ist. Geriatr. Pio Albergo Trivulzio

Laboratorio Analisi

Via Trivulzio 15 - 20146 Milano

Tel.: 02 4029544-403; Fax: 4029549 